\title{
Fictional Characters as Adaptive Systems: The Malaspina and de Graaf Gardens
}

\author{
Angela Ferraiolo \\ Sarah Lawrence College \\ Bronxville, New York, USA \\ aferraiolo@sarahlawrence.edu
}

\begin{abstract}
This paper describes an artwork titled Nine Gardens. The project is a series of slowly evolving digital landscapes meant to represent the interior states of fictional characters. Each garden relies on a system of elements and signals to generate character states then model these as visual elements on screen. In addition to signals from their environment, the elements that make up each garden rely on internal schemes of deliberation to decide their appearance. Deliberation rules were conceived of as 'character states'. The aim of the project is to portray character as a dynamic system or post-dramatic construction as opposed to the conventional view of character as an aggregate of wants and goals. This paper describes the design of two of the systems in the series, the Malaspina and de Graaf gardens.
\end{abstract}

Postdramatic. Agent. Character. Complex adaptive system. Garden. Landscape.

\section{INTRODUCTION}

Nine Gardens is an attempt to model some of the principles of Chinese landscape painting with a dynamic system. It aims for a postdramatic exploration of character through the creation of several small computational gardens. These gardens are made up of agents or elements that both influence and respond to their environments. Like the literati of the Yuan dynasty, the gardens privilege the expression of interior thought and subjective experience over direct representation. Like agent-based systems, they seek identity through process and adaptation, which are expressed on screen as visual strategies of composition. In a postdramatic conception of character, the gardens represent identity as a process, a continually synthesized way of being in a world rather than an actor's initiation of a series of actions in pursuit of a goal. A screenshot of one of the projects, the Malaspina garden, is shown in Figure 1.

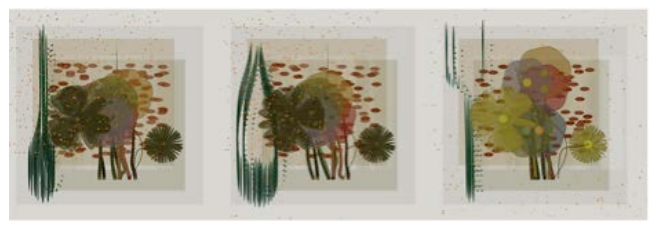

Figure 1: Malaspina Garden at time $t_{0}, t_{1}, t_{3}$

\section{CHARACTER AS LANDSCAPE}

The representation of character through a depiction of landscape has a long history in the visual arts beginning with the oldest visual images known to historians and continuing through works of the Old Masters, academic painters of Europe and the Americas, modernists, abstract artists, postmodernists and even the video game designers of the present day. In Asia, and in China in particular, landscape was an important thematic for all painters. As early as the Tang dynasty, landscape painting allowed Chinese artists a way to hypothesize ideals of character such as optimism, inventiveness, wisdom, or stability. Later, the design and composition of landscapes often served as a metaphor for the social order of more regulated states (Bush 2012, Cahill 1982).

\subsection{The literati painters}

From about the end of the tenth century through the Five Dynasties of the 16th century, one particular group of Chinese artists used landscape painting as an opportunity for the subjective treatment of reality. These 'scholar painters' or 'literati', believed painting, poetry, and calligraphy were closely aligned and sought visual compositions that might be as expressive as language. Literati painting, also known as 'mind 
landscape', rejected the Chinese schools of direct observation and representational technique and aimed instead for the communication of character, point of view, and opinion. For scholar artists, the use of landscape was metaphoric - a collection of shapes and forms presented as conduits of personal judgment (Fong 1973, Lee \& Wai-kam 1968).

During the Yuan dynasty, when China was under rule by the Mongols, some literati rejected court painting due to politics as well as aesthetics. For artists of this period, the rejection of the representational image was also viewed as a protest of China's occupation. Some literati painting, became a mute but tangible form of political dissent. As a group, the literati were male, wealthy, and social elites. These privileges, along with the stance of 'artist', perhaps contributed to the self-confidence they showed in critiquing the court.

The visual techniques of the literati vary from artist to artist but some generalisations have been made. For example, to express agreement with social conventions of the past, literati painters might quote the symbols, motifs, or brushstrokes of the old masters. To express divergence from a traditional view, artists might break academic rules of representation through the use of multiple vanishing points, the clumping of marks in ambiguously composited shapes, the layering of ink to express texture, or the creation of completely new and iconoclastic marks.

Holding broad observations like these in context, it's often better to view the literati individually and to see their techniques as expressions of personal temperament. The great literati master Zhao Meng Fu studied Chinese masterworks with intensity but liked to present himself as a simple and improvisational painter. His works are notable for fast easy brushstrokes that seem improvised and immediate. As a counterpoint, the artist Ni Zhan, who was ruined by taxes, lost all his possessions, and turned to wandering through the countryside, expressed his withdrawal from society through an austere minimalist style that depended on expanses of blank space and dry, withered strokes of a broken brush. A contrast to Ni Zhan would be the painter Wang Meng, who was not opposed to slipping in and out of court life as needed, and who created landscapes full of turbulence, cliffs, caves, and traps all brought to life through colourful, extravagant brush strokes piled one on top of another in dense, rhythmic motifs.

\subsection{Complex adaptive systems}

In our own time, the ideas of the Chinese literati have some resonance with generative artists. Through the practice of generative art, metacreation, and complex adaptive systems, computational artists have adopted algorithmic strategies to create dynamic representations of landscape as well as other visual forms. These procedural works move the image away from representation to emphasise qualities of process. They extend the aesthetic qualities of visual composition to time and motion, but also to simulation, behaviour, and deliberation (Kirsch, et al. 1988, Xiong \& Kang 2016).

The paradigm of complex adaptive system (CAS) has been used many times to simulate plant growth, animal behaviour and other natural phenomena. A skeletal description of a CAS might be a model in which a perfect understanding of that model's individual elements does not predict or give understanding to the behaviour of that model as a whole. A common example of a CAS is a flock of birds where each bird follows an individual flight path thereby contributing to elaborate group behaviours despite the absence of any top down control of trajectories. Epidemics, wars, and economic markets have all been modelled as adaptive systems, a paradigm that can be reductive while remaining expressive.

While there are no single universally accepted definitions for computational emotion, belief, or affect, one type of CAS known as the BeliefDesires-Intention (BDI) model allows for the simulations of agent cognition through the use of symbolic representations of that agent's interior processes. Through BDI it is possible to represent a fictional character's mood or temperament as a number of small elements grouped by a set of rules in operation over an environment. The agent's beliefs are what it knows of its environment. Its desires are its goals, subgoals, or targets. Intentions can be thought of as each agent's level of commitment to the goals to which it is currently subscribed (Puica et al. 2013, Gentner \& Albert 2014). Minsky framed a conception of this type of system as a diverse collection of small entities called micro-agents, small pieces of code that perform specific tasks which are then combined, linked associatively, or sequenced causally into larger cognitive processes which are in turn at work in an environment that provides updates to the system's knowledge base, a so-called 'society of mind' (Minsky 2007).

Generally then, a BDI architecture could model a character's inner states as an adaptive system that allows for inputs from an environment (perception), information processing (deliberation), ways of jurying competing goals (intention), some means of outputting to the environment (action), and the regulation of these processes (metacognition). Framing this model within an art historical context 
of landscape painting asks viewers to view these processes aesthetically.

\subsection{Postdramatic character}

Traditionally, a character represents both internal temperaments as well as their cultural context. Characters overcome weaknesses to pursue goals and progress through a series of dramatic events in service of a super-objective. But since the 1930s, and especially from the 1970s to 1990s, the relationships between character, plot, and narrative have been under experiment and the deconstruction of all literary structures has undergone a large number of innovations including the fragmentation of time, place, and point of view, anonymous or ambiguous discourse, the creation and construction of 'speech surfaces', and the abstraction of texts to the point where groups of entities may represent single narrative movements - in other words, texts in which there seems to be no definable characters at all. At the same time, new forms of media have expanded definitions of character to consider character as network, character as telepresence, and character as modified by user input, behaviour, or simulation.

In this project, character has been imagined as a dynamic process or the ongoing computations of states. Character is not a fixed set of strengths and weaknesses set against several obstacles in pursuit of some final outcome, but an ongoing strategy of perception, computation, adaptation, and response. This conception of character is not separate from more conventional views, but it emphasises character as a pattern of reactions rather than a force acting within a field of possibilities (Fuchs 2012, Lehmann 2006, Sugieria 2004).

Experiments like these are often grouped under the heading 'post-postmodern' or 'postdramatic'. Some are technically accomplished and stylistically elaborate, but the hope is that these explorations amount to more than aesthetic poses. The postdramatic also asks audiences to reconsider the design of character in relation to literary theory. As the meaning of character evolves, so may the significance of our cultural experience.

\subsection{Related works}

The designs that informed this project are early examples of simulation art. These include Karl Sim's foundational experiments with evolving virtual creatures (Sims 1991), Hutzler, Gortais, and Drogoul's work on associating abstract painting with agent-based systems (Hutzler et al. 1999) and Sommerer and Mignonneau's experiments with complex systems and origins-of-life theories
(Sommerer \& Mignonneau 2002). The idea of using environmental signals for communication and for using rule tables for agent deliberation was inspired by several engineers and artists, but especially by the works of John Holland (Holland 1990, 1995, 2012), Jon McCormack (McCormack 2001, 2005), Gordon Munro (Munro 2016), and Peter Beyls (Beyls 2016). Jon Elster's writing on political psychology (Elster 1994), as well as the social psychology concepts of self as noted by Epstein (Epstein 1999), Kassin (Kassin, et al. 2007), and Miller and Page (Miller \& Page 2009) gave the basis for simple, reflexive character states that could be modelled as systems. Wen Fong's history of landscape painting during the Yuan dynasty (Fong 1973) influenced some of the visual strategies of the gardens, as did the art historical writings of James Cahill and Susan Bush (Bush 2012, Cahill 1982). Ideas for the procedural design of characters were informed by writings in literary and dramatic theory from Fuchs, Lehmann, and Ryan (Fuchs 2012, Lehmann 2006, Ryan 1991).

\section{THE MALASPINA AND DE GRAAF GARDENS}

This paper describes the design of two of the nine systems in the series, the Malaspina and de Graaf gardens. Malaspina is imagined as a mid-career naval officer who seeks intense experience but values stability. Internally, he is divided between a desire for change and respect for tradition. The de Graaf garden visualises a female persona that is driven to build a financial empire but whose ambitions tend to digress into childlike fantasy. Brittle and extreme, the de Graaf persona shifts rapidly from a demanding outward intensity to quiet and contemplative introspection.

\subsection{Design concept}

The following decisions helped structure the artworks: Each agent gives form to one of the visual elements that make up the garden as a whole. Elements don't aspire to goals and don't pose dramatic questions of action or destiny, but they do have states they want to maintain or opposing states they want to enter. Agent identities are rulesets, which, in the tradition of landscape as metaphor, were written to define character in terms of shape, colour, motion, and behaviour. Elements can perceive their environment and adapt to change either individually or collectively. Depending on the procedural nature of a character, some agent rules are fixed while other rules are mutable. Although elements have some autonomy when making internal revisions, they do not have the ability to deviate from their rulesets completely. Instead, rules are intended to foster the creation of a range of visual responses coherent to their owners. The overall structure of a landscape system is shown in Table 1. 
Table 1. System Diagram

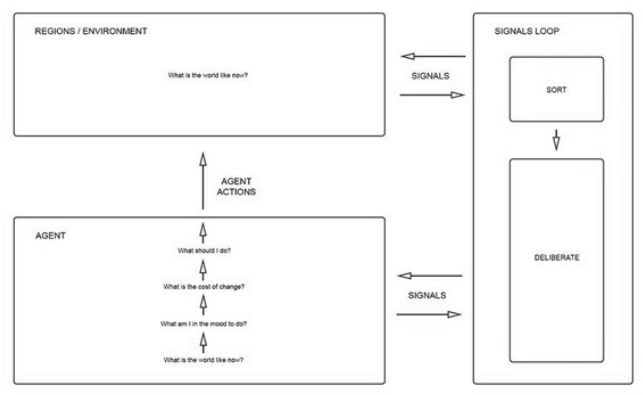

\subsection{Visual considerations}

For the most part, Malaspina endeavours to meet his understanding of his role as an officer. However, success in this aspect of his self-view requires the choice of character states that might be disruptive and at times encourage a reevaluation of priorities before choosing an action. These interior conflicts are expressed through the juxtaposition of several formal qualities in the garden. For example, a restrained use of muted colour signals Malaspina's turn towards tradition while a choice of experimentation would be denoted by a brighter, more impulsive use of colour

In addition to these colour narratives, the Malaspina garden also uses scale, rate of change, motion, foreground and background placement, sparseness, density, thicks, thins, and edge complexity or simplicity to express the following oppositions at work within the character:

\section{Elegance versus Vulgarity \\ This opposition represents Malaspina's diversions in aesthetic tastes from the austerity of his military training to the bright, cheerful energies of ordinary society. The dichotomy is meant to tie the ideological and political tensions at work within the character to visual expressions of line and colour.}

\section{Likeness versus Abstraction}

This opposition relates Malaspina's persistent doubts concerning the spiritual and the rational, the imaginary and the practical. He is an agent who continually struggles with the relationship between the natural and the designed.

\section{Surface versus Depth}

An opposition between the dry, hard style of high definition and a softer, less obvious approach to form and line. This dichotomy represents Malaspina's simultaneous attractions to the stability of tradition while admitting the necessity of change.
The de Graaf garden uses a similar design to explore different conflicts. The personality driving this garden shifts from the need to attract the outside world and draw it closer versus a digressive instinct to escape the demands of reality by turning inward. The transitions produced by this structure of self include reversals of form and shape, colour inversions, and shifts in composition as a way of representing the conflicts that define this character:

\section{Practicality versus Fantasy}

This conflict describes the persona's impulse to control her world while feeling the need to retreat from external demands. The conflict is represented by inversions of form in the main elements of the landscape.

\section{Ambition versus Self-Doubt}

The persona's ego goes through frequent shifts between self-confidence and lack of faith. There is an impulse to succeed followed by a crisis of resolve. Expressed through speed and colour.

\section{Action versus Calculation}

This persona's need to take action is often in opposition to her tendency to analyse Expressed through the smaller elements of the system, these tensions are described by complications in form which move gradually from the expected and regular to the idiosyncratic and the somewhat bizarre.

As in the Malaspina garden, the de Graaf landscape can appear disjointed, conflicted, and lacking in harmony, while at other times seeming ordered and serene. These oppositions do not signify confusion as much as they show character as process and the individual as a malleable, evolving state space

\section{SYSTEM DESIGN}

Based on these aesthetic conceptions, the gardens in the series were constructed from two kinds of classes that send and receive signals through a main control class. Garden elements or elements change over time by receiving signals, deliberate, choose and execute actions, and send signals. Regions hold the global state of the system and through information on the global state can influence elements.

\subsection{Elements}

There are five main groups of elements in a garden system: shapes, lines, columns, patterns, and textures. Depending on the deliberations of elements, these elements are used individually or repeated, placed centrally or overlapped. Examples of the garden elements in the Malaspina garden are shown in Figure 2. 


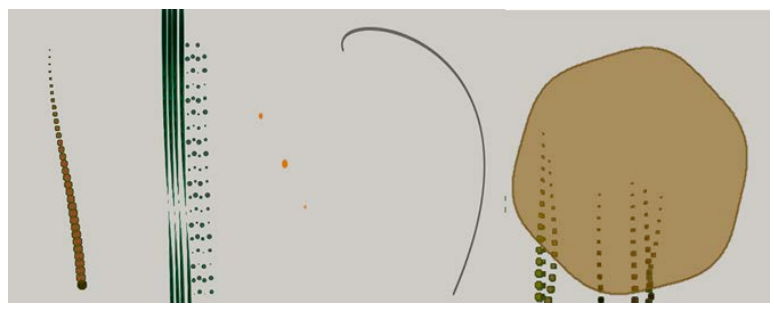

Figure 2: Elements of the Malaspina Garden

\subsection{Regions}

Certain elements are also assigned to the system as regions and computed as region rather than character elements. Time moves forward at the same rate across all of the elements in a garden in discrete steps. Regions are used as system time keepers and are programmed to create global states of seasonality: summer, fall, winter, spring. Both the Malaspina and de Graaf gardens contain two background fields or 'washes' and at least one background element or 'curtain' that are computed as regions. A chart of garden regions is shown in Table 2.

Table 2. Garden Regions

$\begin{array}{ll}\text { Region } & \text { Upper Region } \\ \text { Elements } & \\ & \text { Lower Region }\end{array}$

Regions can contain multiple garden elements, shapes denoting flowers, leaves, weeds, or more abstract organic forms that echo these forms. Initially, elements are placed within regions randomly then progress according to deliberation.

\subsection{Signals}

Signals are simple integer values sent from regions and elements through a main signal loop where they are processed as deliberations. Region signals determine the season and overall temperament of the system. Element signals are matched to a table of rules sensitive to the context of the system's overall state. During large shifts in state, a third sent of signals can be issued by the control class. These signals allow elements to choose with some degree of autonomy to continue their present rule set or shift to another set of rules within a master table. A partial list of agent and environment signals is shown in Table 3 . Signals.

Elements can receive signals describing certain global states in the system such as season, current character state, or target character state. Elements can also receive signals about specific visual events and actions as well as changes in the states of other elements and garden elements that might affect their current or next immediate goal. There can be as many as twenty signals active in the garden at any time.

Table 3. Signals (Partial) - Malaspina Garden

$\begin{array}{lll}\text { Object } & \text { Sends } & \text { Receives } \\ \text { Region } & \text { Season Marker } & \text { Season Marker } \\ \text { Region } & \text { Main Character Wt } & \text { Main Character Wt } \\ \text { Region } & \text { Opposing Character Wt } & \text { Opposing Character Wt } \\ \text { Region } & \text { Rate Of Change } & \text { Rate Of Change } \\ \text { Region } & \text { Lifecycle Markers } & \begin{array}{l}\text { Energy } \\ \text { Element }\end{array} \\ \text { Element } & \text { Main Character Wt } & \text { Season } \\ \text { Element } & \text { Opposing Character Wt } & \text { Opposing Character Wt } \\ \text { Element } & \text { Main Character Wt } & \text { Main Character Wt } \\ \text { Element } & \text { Opposing Character Wt } & \text { Opposing Character Wt } \\ \text { Element } & \text { Shape Shifts } & \\ \text { Element } & \text { Rates Of Change } & \end{array}$

Character states are constant over each time step, but intensity, stability, and decay weights may influence their duration and stability. Deliberation results in a recalculation of current and target character states at each time step.

\subsection{Actions}

An important goal of the artwork is to move away from a conception of character as the workings of and individual pursuing a want through a superobjective to a view of character as a network of processes that manage an ongoing set of states designed to be in conflict with one another. In this kind of model, character is managed through deliberation and events and processes are readable through the visual representation of agent response in the environment. Elements can potentially perform a number of visually readable actions including moving, turning, changing shape, changing colour, and changing speed or direction.

Once an action is undertaken, an agent signals the event back to the environment and the weight of the event is calculated as an input to the global state of the system and to relevant agents or elements.

\section{DELIBERATION}

In a familiar design scheme, signals handle all the communication in the garden. A control class takes in messages from agents and regions, processes them, then sends response messages back out. Regions use these signals to update the global states of the garden. Elements receive this signals, process them according to their current character states, and enact an action or visual change in the environment through their properties. Elements send messages describing their actions and these messages serve as the starting point for the next cycle in a garden's development. 


\subsection{Personality}

In each garden, reactions are modelled on the concept of schema or, very generally, a mental construct describing the way an individual organises their view of themselves and their identity in the world. Schemas can provide a framework for the ways an individual categorises ideas and emotions. In changing environments, schemas can organise perception by quickly sorting new information into pre-existing domains of knowledge that to varying degrees, constitute a personal definition of self. For example, an individual who sees themselves as 'social' would receive information of their world within the context of a sociable schema and would process that environmental information accordingly, while a person with a more interior focus would almost certainly process those same perceptions differently. In addition to organising an individual's beliefs about the world and themselves, schemas also influence the ways an individual receives new information and how new information is understood and acted upon.

As a character who is simultaneously attracted to tradition and experimentation, Malaspina values conservation of identity while simultaneously feeling attractions to nearby events and changes in environment. Malaspina seeks intensity while trying to maintain security. With these tendencies in mind, a character self-schema was modelled across four categories: stimulation, conformity, mastery, and egalitarianism. Malaspina character states and their modifiers are shown in Table 4. Malaspina Garden - Personality.

Table 4. Malaspina Garden - Personality

$\begin{array}{lllll}\text { State } & \text { Intensity } & \text { Sociability } & \text { Stability } & \text { Decay } \\ \text { Stimulation } & \text { high } & \text { high } & \text { low } & \text { fast } \\ \text { Conformity } & \text { low } & \text { low } & \text { high } & \text { slow } \\ \text { Mastery } & \text { high } & \text { low } & \text { high } & \text { slow } \\ \text { Egalitarianism } & \text { high } & \text { high } & \text { high } & \text { slow }\end{array}$

The de Graaf persona exhibits bigger and more rapid shifts in character. de Graaf rushes to engage her environment, then suddenly retreats. Her schema is designed as a pair of outward facing states set in opposition to a pair of inward facing states, or risk and engagement versus calculation and withdrawal. The de Graaf character states and their modifiers are shown in Table 5. de Graaf Garden - Personality.
Table 5. de Graaf Garden - Personality

$\begin{array}{lllll}\text { State } & \text { Intensity } & \text { Sociability } & \text { Stability } & \text { Decay } \\ \text { Risk } & \text { high } & \text { high } & \text { low } & \text { fast } \\ \text { Calculation } & \text { high } & \text { low } & \text { high } & \text { fast } \\ \text { Engagement } & \text { high } & \text { high } & \text { low } & \text { fast } \\ \text { Withdrawal } & \text { high } & \text { low } & \text { high } & \text { fast }\end{array}$

\subsection{State weights}

Each agent is initialised with starting values for all four character states and their modifiers. The two highest values determine which states have the greater influence over the agents choices and properties. Deliberation either matches the set of weights produced at a specific time step to a character's schema or set of states and transition rules. Deliberation can keep a character in its current state or push a character to an alternate state.

Before state weights are matched to rules, they are subject to the influence of three modifiers: intensity, sociability, stability, and decay. Intensity modifies how much of an environmental signal survives passage to agent deliberation. Sociability modifies these weights again based on a character's willingness to receive inputs. The stability modifier makes it more or less costly to change from one state to any other state. The decay modifier means some messages can reside in a signal list longer than others.

For example, an agent in a state of conformity might have a low modifier weight for character intensity. If a signal from the environment should enter the signal loop and be deliberated as a decision to seek a state of higher stimulation, that agent might execute an action that increases system intensity. However, as in the case of a move towards stimulation, that higher intensity rate can fade quickly due to its fast decay modifier. It may take more than one attempt at stimulation to overcome the stability modifier on conformity. Another time step and another round of signalling would need to continue this signal for it to successfully change deliberation. An example of this would be the rate of change in the de Graaf garden as compared to the rate of change in the Malaspina garden. The de Graaf garden exhibits low decay rates across all of its character states making it likely to change more rapidly than Malaspina.

\subsection{Signal and action control loops}

The events that cause agents to recalculate their states are messages or signals, which are continually being sent and received to and from elements and from elements to and from the environment. All signals pass through a main signal 
loop where they are processed before being relayed to back to elements and regions. Regions are simple reflex entities, but both the signal loop and element classes can process messages.

Processing can consist of sorting, rule matching, or calculation. Taken together these computations are called deliberation. Generally, the process is as follows:

The signal loop receives signals from elements and the environment. The most basic message would be a signal of a new time step. Messages can also be notices of region events, changes in the state of the world, changes in the state of an element, etc.

The signal loop sorts messages according to an internal representation of the whole garden as well as a representation of individual agent tendencies. If at the time of its receipt a signal protocol is not in the list of the signal loop rulesets, the signal is disregarded and no further action is taken. If the signal successfully matches one or more of the rules in the table, the loop signals the relevant garden elements and regions.

Depending on their current character states, elements respond to signals with varying degrees of compliance or resistance. In addition to the rules found in the signal loop, each element has a second, individual table of rules.

Elements may respond autonomously. In some cases, agents may give signals an unforeseen or idiosyncratic response, that is, under some conditions agents may exhibit a degree of autonomy.

After processing signals, elements update their methods and properties.

All element and region processes carry an energy decrement. The amount of energy an element expends on an action response also depends on its character state. Depending on an agent's mood, processes may be more or less expensive. An element can die if its energy weight falls below zero. An element can spawn if its energy weight is greater than one.

\section{IMPLEMENTATION}

So far this project has gathered ideas from a number of disciplines in the hopes of assembling an adaptive system that might model character. What follows is an example of how these pieces can fit together to create a changing digital landscape.
Initialisation

A season, which determines the garden's initial global colour palette and sets the energy limits of some agents, is chosen at random.

\section{Configuration}

Initialisation weights are used to determine the garden's starting configuration. This starting configuration determines the initial rendering of a garden.

\section{Region Generation}

According to the global season definition, the canvas is filled with a uniform background colour. Regional patterns that express global properties like global energy, overall character state, etc. are also sent to the screen.

\section{Element Generation \\ Using weights calculated in initialisation, elements are generated and rendered to the screen. A system clock is started.}

\section{First Time Step}

Signalling starts. The first set of messages is sent from agents and regions to the main control class.

\section{Signal Loop}

The signal loop gathers region and agent messages and matches these to character state rules. New messages are sent to agents and regions describing the current states of agents.

\section{Deliberation Loop \\ Elements receive signals from the signal loop and match these to their internal rulesets. Elements adjust their properties accordingly.}

\section{Render Loop}

New visual renderings are updated on screen.

The system continues, with states and visuals continually recalculated. Future seasonality is influenced by global energy values, but follows a conventional calendar of summer, fall, winter, and spring.

\section{RESULTS}

This section presents several screenshots of the Malaspina and de Graaf gardens as they are computed, though a better understanding of the project would come from watching the environments under computation as they go through their simulations. On a similar note, unlike the results expected of a sociological or statistical model, the results gathered from a system intended to produce an artwork aren't expected to provide insights on a specific question as much as they are expected to give evidence of an intended aesthetic result. 
The figures below were captured to give a sense of the appearance of these gardens at the extremes of their character states. State extremes are typically the points where a system might produce surprises or novelty in their appearance.

For these pictures, both gardens were initialised to autumn as their season start. Their four character states were started in balance with equal weights of $0.25,0.25,0.25,0.25$. Modifier weights for intensity, sociability, stability, and decay rate were initialised as 0.25 (low) and 0.75 (high) according to each character's internal schema (Tables 4 and 5) and both gardens were initialised with at least four elements or garden agents that illustrate character state. Figure 3 shows the Malaspina garden in season fall with its four character states of stimulation, conformity, mastery, and egalitarianism equally weighted, in other words a character in balance.

At his most conservative, Malaspina would be receiving signal information that pushes elements away from stimulation and egalitarianism towards mastery and conformity. The overall stability of the garden would be high. Elements would appear to simplify their form and moderate their ranges of hue and saturation. Regions and elements across the system would need significant motivation to change and their rate of change would be slower than average. An example of an extreme state of Malaspina's conforming tendencies is shown in Figure 4.

Based on different signal information, Malaspina would move in a different direction. For example, the system would under other circumstances push away from conformity and towards stimulation. The overall stability of the garden would decrease. Elements would complicate their shapes and intensify in hue and saturation. The system would tend towards change and its rate of change would be faster than average. A screenshot of an extreme state of Malaspina's adventure seeking tendencies is shown in Figure 5.

Figure 6 shows the de Graaf garden initialised to autumn with its four character states of risk, calculation, engagement, and withdrawal equally weighted. Figure 7 shows that same garden as it moves towards a character state of risk seeking, faster change, and less stability. In Figure 8, de Graaf has moved towards a state of withdrawal. Its forms are inverted and its colours are muted. At this point, the garden tends toward stability, but in keeping with the impulsive nature of its character, even this extreme has a fast rate of decay meaning signals enter and leave deliberation at a faster rate.

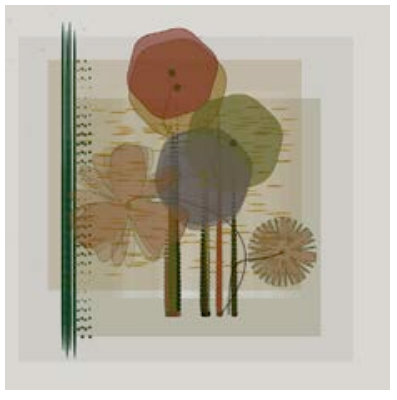

Figure 3. Malaspina - Character State: Balanced

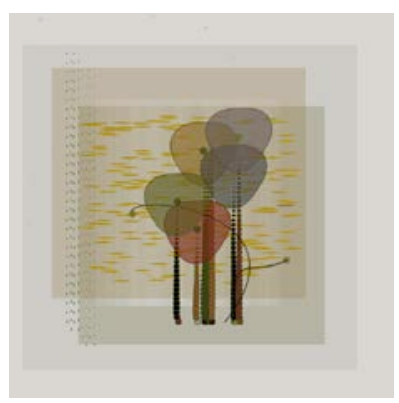

Figure 4. Malaspina - Character State: Conforming

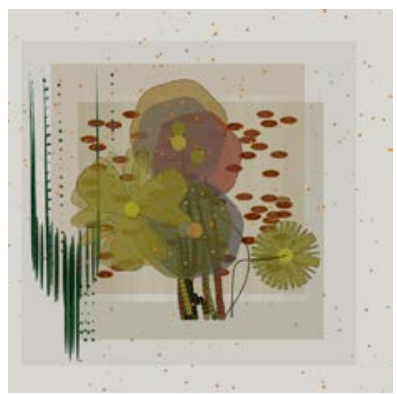

Figure 5. Malaspina - Character State: Stimulation

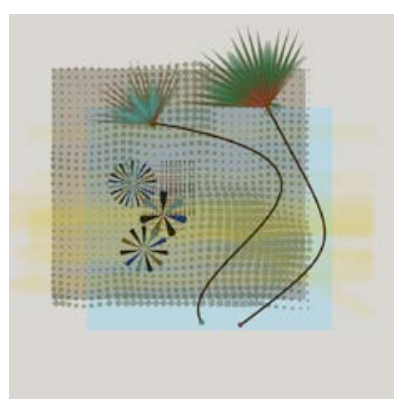

Figure 6. de Graaf - Character State:Balanced

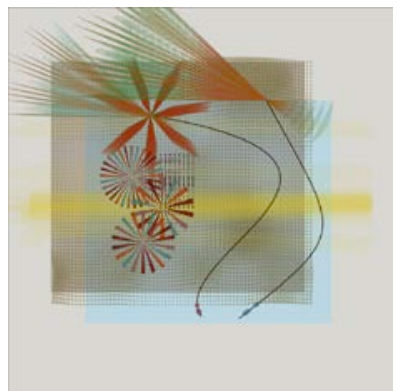

Figure 7. de Graaf - Character State: Risk Seeking 


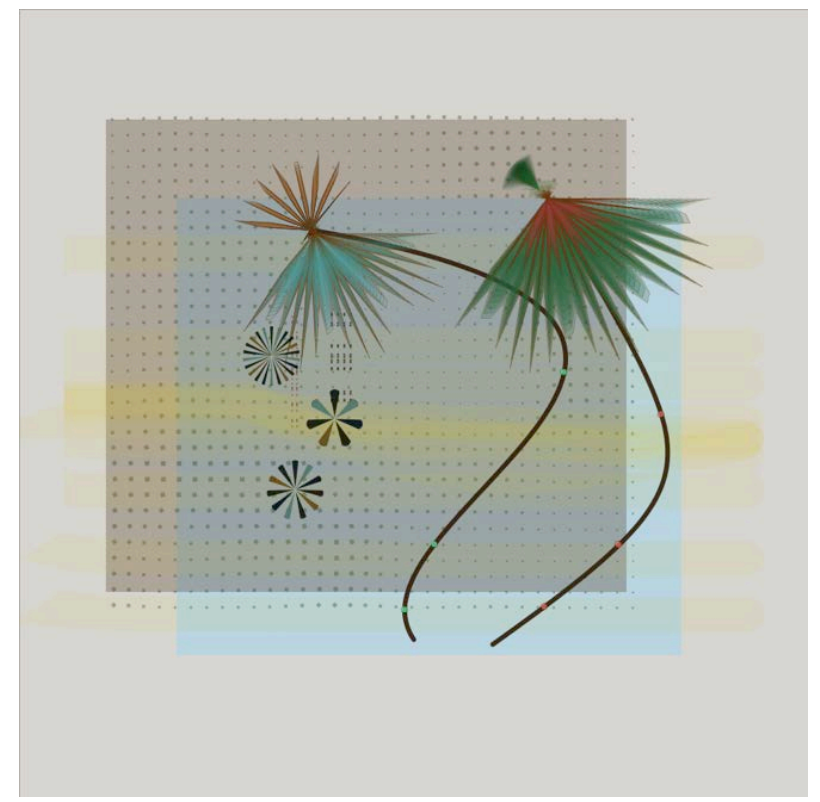

Figure 8. de Graaf - Character State: Withdrawing

\section{CONCLUSION}

This paper described an artwork that uses CAS to simulate character. The project draws on ideas from the Chinese literati painters of the Yuan dynasty, agent based adaptive systems, and postdramatic literary theory. The motivation for this project was to explore character as an adaptive system as an alternative to the view that a character is a dramatic entity with a super-objective that seeks a goal. Gardens were designed according to conflicting character states. As these gardens were computed, they exhibited changes in colour, shape, and composition that could be thought of as visual identities.

As an initial experiment this project aimed for simplicity. There are significant limitations in its design. Agent deliberation could be expanded to include ways for elements to acquire and evolve beliefs. Elements could also be designed to use main and opposing character weights as building blocks for planning and strategy. However, even in this simple example, it seems character is can be expressed by a system and that it is possible to model character dynamically.

\section{REFERENCES}

Beyls, P. (2012) Autonomy, influence and emergence in an audiovisual ecosystem. In Proceedings of the Generative Arts Conference, Rome, Italy.

Bush, S. (2012) The Chinese Literati on Painting: Su Shih (1037-1101) to Tung Ch'i-Ch'ang (1555-1636). Hong Kong University Press.
Cahill, J. (1982) The Compelling Image: Nature and Style in Seventeenth-Century Chinese Painting. Vol. 1979. Harvard Univ Pr.

Elster, J. (1993) Political psychology. Cambridge University Press.

Epstein, J. M. (1999) Agent-based computational models and generative social science. Complexity 4(5): 41-60.

Fong, W., and Marilyn, F. (1973) Sung and Yuan paintings. Metropolitan Museum of Art.

Fuchs, E. (2012). The Death of Character: Reflections on Theatre after Modernism. Indiana University Press.

Gentner, D., and Stevens, A. L. (2014) Mental models. Psychology Press.

Holland, J. H. (1990) Echo: Explorations of evolution in a miniature world.

Holland, J. H. (1995) Hidden order how adaptation builds complexity. No. $003.7 \mathrm{H} 6$.

Holland, J. H. (2012) Signals and boundaries: Building blocks for complex adaptive systems. MIT Press.

Hutzler, G., Gortais, B., Drogoul, A. and Hill, B. (1999) Garden of Chances. Leonardo 32(3): 181-182.

Kassin, S., Fein, S. and Markus, H. R. (2007) Social Psychology: Readings. Wadsworth Publishing Company.

Kirsch, J. L., and Kirsch, R. A. (1988) The anatomy of painting style: Description with computer rules. Leonardo(1988): 437-444.

Lee, S. E., and Wai-kam, H. (1968) Chinese art under the Mongols: the Yüan dynasty, 1279-1368. Cleveland Museum of Art; distributed by the Press of Case Western Reserve University.

Lehmann, H. T. (2006) Postdramatic theatre. Routledge.

McCormack, J. (2001) Eden: An evolutionary sonic ecosystem. In: European Conference on Artificial Life, pp.133-142. Springer, Berlin, Heidelberg.

Miller, J. H., and Page, S. E. (2009) Complex adaptive systems: an introduction to computational models of social life. Princeton University Press.

Minsky, M. L. (2007) The Society of Mind. New York: Simon \& Schuster Paperbacks.

Munro, G. (2016) Nanocosm, Art exhibition, 19th Generative Art Conference, Florence, Italy.

Puica, M. A., and Florea, A. M. (2013) Character beliefdesire-intention agent model: Previous work and proposed architecture. International Journal of Advanced Research in Artificial Intelligence 2(2) (2013): 1-8.

Ryan, M. L. (1991) Possible worlds, artificial intelligence, and narrative theory. Indiana University Press.

Sommerer, C., and Mignonneau, L. (2002) Modeling the emergence of complexity: Complex systems, the origin of life and interactive on-line art. Leonardo 35(2): 161-169.

Sims, K. (1994) Evolving virtual creatures. In: Proceedings of the 21st annual conference on Computer graphics and interactive techniques, pp.15-22. ACM.

Xiong, L., and Zhang, K. (2016) Generation of Miro's Surrealism. In: Proceedings of the 9th International Symposium on Visual Information Communication and Interaction, pp.130-137. ACM. 